them is determined."* Dr. Hughes Bennett has admirably demonstrated the modus operandi of the capillaries in exudations. $t$ In a valuable work of Dr. W. Addison, the power colourless cells exert in controlling the nutritive changes is, I believe, correctly explained. While under abnormal action there is frequently an excess of cells, and they are diffused among parts adjacent $; \ddagger$ I mean beyond their natural boundaries.

The present communication may be considered as an humble introduction to a more extended research into pathological hæmatolgy. I am happy to say Mr. Ancell is resuming the same subject in a series of able lectures in the course of publication; $\S$ and it is impossible to orerrate the importance of this branch of morbid anatomy. On reflection, we cease to wonder that diseases arise from modifications of the blood, when we take but a distant view of the numerous circumstances by which it may be altered. Atmospheric vicissitudes, aliment, drinks, respiration, and the condition of the nervous system, by which all the secretions are governed, exert a powerful influence on the blood, and produce numberless changes in the constitution. There would be a great advantage in having a more accurate mode of gauging the serum and crassamentum, since it would furnish some index of health and its deviations. The older authors were of opinion that the source of many disorders originated in the blood, but there is a great vagueness in their notions, for they were unacquainted with quantitative, physical, or chemical analysis. It has been reserved for modern physiologists to weigh and measure those realities which were before only matters of conjecture. Further inquiries are necessary to determine what value may be attached to the theory of disintegration of the blood. Of this I feel assured, we are advanced far enough to enable us to draw the conclusion that organic changes of the solids in general are not antecedent to an isolation of the elementary organisms, but depend on the dispersed and abnormal existence of the circulating corpuscular fluids. Hence the science of hæmatology deserves our highest consideration, and must be eventually estimated as the very foundation of all pathological investigation.

The examination of the blood is often desirable while the patient is under medical treatment; and in postmortem inspections it should always be attentively noticed.

- Outline of Pathology, p. 120.

+ Monthly Journal of Science, Jun., 1848.

\$ Physiological Researches.

- Commencing in No. I., (new series,) of the Medical Times.

MM. Bequerel and Rodier have lately presented a memoir to the Academy of Medicine, "On Anæmia from Diminution of Albumen, and che Dropsy consequent thereon."

\section{CASE OF OVARIOTOMY.}

By GEORGE NORMAN, Esq., F.R.C.S.,

SENIOR-SURGEON TO THE BATH UNITED HOSPITAL.

Read at the Quarterly Meeting of the Bath and Bristol Branch, Dec. 19, 1850.

Elizabeth North, aged 23, enjoyed good health until March of the present year, when she began to have a disturbed state of stomach, pains extending over the abdomen, irregular menstruation, and in May she became sensible of a swelling on the right side of her body. She became the patient of Dr. Davies at the United Hospital, who has obliged me by furnishing the following particulars :-

" Elizabeth North was admitted into the United Hospital under my care on the 7 th of June, and was discharged on the 2nd of July. At the time of her admission she was suffering much from derangement of her general health, and the menstrual function had been out of order for some months. At this time the tumour on the right side of the abdomen did not appear to be larger than an orange; no doubt was entertained of its being ovarian. The treatment was addressed entirely towards the improvement of her general health, and she quitted the hospital much improved in that respect, the tumour remaining the same."

She was admitted into the hospital again, under my care, on the 19th of September. The tumour then appeared to be nearly as large as the head of a child at birth; it was quite moveable in the abdomen and appeared to be attached below by the broad ligament of the uterus, for no doubt was entertained of its being a tumour of the right ovary. It was firm to the touch and gave no sense of fluctuation; her general health had much given way; she had pain extending over the abdomen, and at times severe cramps in the bowels; a fold of vagina protruded full two inches beyond the external pudendum, even when in the recumbent position, and she had endeavoured in vain to keep this up by a pessary before her admission. It was with great difficulty the os uteri could be felt; after a considerable examination it was found under the arch of the pubes. She was kept in bed, took iodide of potassium, and used iodine frictions to the abdomen, still the tumour increased in size and the pains in intensity.

The case was considered by my colleagues and myself, to be an ovarian tumour, and that if ovariotomy were ever advisable, this patient's case was one calling for it. The prolapsed state of the vagina, the weight of the tumour, and the continued pain, rendering it impossible for her to do anything for her support; the tumour was increasing and there seemed to be no hope of its growth being arrested. It was agreed that she should discontinue all remedies for a fortnight. At the end of that time her general health had not improved and the tumour appeared to be as large as the impregnated uterus at the fourth, or between that and the fifth month. I then explained to the patient the nature of the disease, told her that an operation had in some 
instances been succesful in the same disease, but that the risk was great and the result doubtful. She replied that she had expected I should make the proposal to her, that she had quite made up her mind, and was ready to undergo the operation whenever I thought proper. A delay, however, occurred from her expecting to be unwell in two or three days, and it was not desirable to operate at or near that period. She became unwell, and after ten days the operation was fixed for the 8th of November. She left off meat for a few days before, and took a mild aperient. Fires were kept for two or three days in the operating theatre, and also in a small ward adjoining it, where it was intended she should remain after the operation, and where she was directed to go to bed the morning of the intended operation.

At twelve o'clock on Friday, the 8th of November, Dr. Davies, who kindly undertook to manage the administration of chloroform, put her fully under its influence in her bed, apart from all those assembled in the theatre adjoining. In a state of perfect unconsciousness she was brought into the theatre, and kept in that state during the whole time she remained there. An incision was made in the linea alba, about five inches in length, commencing an inch above the umbilicus, and avoiding that, it was extended downwards towards the pubes. The periosteum was raised with a pair of forceps, and opened to nearly the same extent; instead of any part of the tumour appearing, several convolutions of the small intestine protruded, these with some little delay were returned into the abdomen with great adroitness, by my collegue Mr. Gore, and retained there. During that time I had ascertained that the anterior parietes of the abdomen adhered very considerably, and firmly, to the tumour on each side of the incision; also that the adhesions below were considerable, and apparently insurmountable, and it was then discovered that a portion of the small intestine, full two inches in length, adhered firmly to the anterior part of the tumour. All hope, therefore, of removing the tumour being gone, no time was lost for the purpose of ascertaining its precise nature, but the integuments were brought together by five common sutures, straps of adhesive plaster were applied, some folded lint placed over with a bandage, and she was removed to her bed in the same state of unconsciousness as when she was brought from it. I saw her four hours after; she was then recovering from the effects of the chloroform, but was suffering from sickness, and pain in the abdomen. Pulse 115, of good strength and fulness. To take one grain of opium directly, and to have nothing but toastwater.

11 P.M.-Still in pain; has vomited the toast-water; pulse 116. To have one grain of opium every four hours.

Saturday, 9 A.m.-No sleep during the night; has vomited the toast-water three or four times; complains of great pain in the abdomen, not, however, increased by pressure, and no tension; pulse the same as last night. I had the satisfaction of hearing that she had no knowledge of having been removed from her bed. or any recollection of the operation. To continue the opium.
4 P.M.-Rather better; pulse 110.

11 P.M.-Has vomited, and is in pain; pulse 116. To continue the opium every four hours.

Sunday, November 10th, 9 A.M.-Has had a little sleep ; had taken a little thin gruel, which was thrown up almost directly; has passed water; pulse 112. To take the opium every six hours. She remained much the same during the remainder of the day. There was some tension of the abdomen, and the bandage was taken off. There was very little pain on pressure.

Monday, 9 A.м. (the fourth day).-Had slept a little; still complains of pain; has vomited twice; has passed urine; tension of abdomen less; pulse 110. To continue the opium.

11 P.M.-She feels better; has vomited but once; pulse 110.

Tuesday, Wednesday, Thursday, and Friday.During these four days she has been getting better, and has only vomited twice; pain less; has only taken the grain of opium about three times in twenty-four hours; pulse from 100 to 110 . Has taken barley-water and gruel.

Saturday (the ninth day).-Still better; pulse 100 ; no vomiting; very little pain; no tension of the abdomen; the sutures removed; the wound partly united, and looking very healthy; bowels not relieved since the operation. To continue the opium, and to have a little weak beef-tea.

Sunday.-Has slept well; pulse 100. To have two ounces of castor oil, which, not operating, was repeated in the evening.

Monday, 11 A.M.-The oil has operated freely; pulse 90 ; the wound healed excepting at two points, the upper and lower end of the incision. From this time she went on well without pain or sickness. Pulse generally about 80 .

On the fifteenth day after the operation it was ascertained that there was no protrusion of the vagina, and it appeared to me on examining the abdomen that the tumour was not of the same size as it was at the time of the operation. The lower part of the wound still remained open, but the quantity of discharge was too small to admit of supposing it came from the tumour. The diminution was supposed to arise from the total abstinence from food for some days, the low diet afterwards, and the constant recumbent position. After this time it appeared to me that the size of the tumour was gradually diminishing, although she was taking animal food, and by the end of November it was quite evident that the tumour was not more than half its former size. An examination per vaginam was made. The vagina was perfectly natural, and the os uteri was found in the ordinary position. The patient continued to improve, she gained flesh, and her countenance regained the healthy appearance. She was, however, kept to her bed, as the lower portion of the wound was not quite healed; soon after, however, it healed, and she was allowed to get up, from which she found no inconvenience, and she was shortly able to walk about the ward with freedom.

On the 9th of December Dr. Davies and I made an 
external measurement of the tumour, and of its position, with the following results:-Distance from the right anterior spinous process of the ilium, to the edge of the tumour, one inch and a quarter; from the left spinous process, to the edge of the tumour, four inches and a half; distance from the umbilicus to the upper margin of the tumour, one inch and a half, (it is to be remembered, that at the operation it was necessary to commence the incision one inch above the umbilicus'; transverse diameter of the tumour fonr inches, vertical diameter three inches and a half; length of cicatrix five inches. The feel of the tumour was harder and more irregular than before.

On the 17 th of December Dr. Davies and I repeated the measurements, and found them to be the same, but she had gained so much in flesh and fulness of the abdomen, that it was not so easy to get the exact measurement. She expressed herself as being quite well, and she left the hospital the following day.

To account for the very considerable diminution in the size of the tumour which has taken place since the operation, is a matter of difficulty, and can only be one of conjecture. It may be that a certain degree of inflammation followed the exposure and the handling of the tumour, (though there was very little of the latter,) and that the vessels of the normal structure of the ovary may have poured out fibrin, and so formed a barrier between the cysts forming the bulk of the tumour and their supply, and this ultimately may have compressed and obliterated them; but be that as it may, the fact of a most important diminution remains, and as the young woman resides in Bath, I shall be enabled to know the more permanent result.

What has occurred in reference to this tumour may throw some light on cases were such tumours have spontaneously disappeared, of which I believe there are many instances. I had an opportunity of ascertaining one recently. A lady whom I had examined several times nearly twenty years ago had an ovarian tumour full as large as the one we have been considering. I saw her a fortnight ago on another occasion, and had the opportunity of examining the abdomen; I could find no trace of the tumour, her account was that the tumour had remained for some years as when I had examined it, that in the last few years it had gradually diminished, that latterly she could only occasionally find it, and that it was very small. I did not find it, but it may be, that being small, it remains for the most part in the cavity of the pelvis, and she described symptoms which made that probable, but it was not admissible to make an examination per vaginam, to investigate it. Another case has been recently told me by Dr. Robert Ferguson, of Park Street, Grosvenor Square, of a lady who consulted him for an ovarian tumour, of considerable size, and in whom, on her returning to him twelve months after, he could find no trace of it. I know another lady, whose abdomen $I$ had frequently examined, who had a similar tumour full twenty years ago. Sir Astley Cooper also examined it, and gave the same opinion, I had given. In this lady, who is now living, it produced nothing more than inconvenience, and if I may judge from the altered appearance of her shape, the tumour must now be very much diminished, but I have had no opportunity of examining it. I have also known an instance where a considerable tumour of the kind formed a communication with the vagina, and after a continued discharge the tumour disappeared, and this person is now living. I know another lady who had for some years a tumour in the abdomen as large as a child's head, which also produced a displacement of the uterus and other distressing and painful symptoms, but which have now entirely ceased, and the tumour became diminished in size. Another instance has been related to me by Dr. Brabent, of a lady who had a large tumour in the abdomen for many years, which always become larger at the time of menstruation, and which gradually diminished after the cessation of that function, and finally disappeared.

I apprehend the case in which I have operated must be placed as opposed to the propriety of the operation, for if in that case it was not possible to foreknow the existence of insurmountable adbesions, I do not know how anything like certainty can be arrived at; and as this patient's life was certainly in jeopardy for three days, one cannot but think such explorative operations must be attended with considerable danger. Add to this the proportion of deaths after the tumours have been removed, and it will appear probable that the balance will turn on that side. The subject, however, is now fully and perfectly under the consideration of the profession, a great mass of information has been gained, and more will no doubt, for it is to be hoped that every case, whether successful or otherwise, will be recorded, and also those cases of ovarian tumours which have remained harmless, as well as those which have proved fatal, and then we may expect from our profession a dispassionate and correct conclusion.

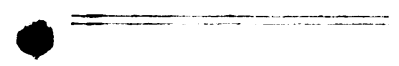

\section{ON THE COMPARATIVE SIZE OF THE HAND OF THE ACCOUCHEUR AND OF THE FEMALE PELVIS.}

By GEORGE KING, Esa., SURGON-ACCUCHEOR TO THE DOBCAS SOCIRT, BATH.

The following observations on the size of the hand and arm in the practice of midwifery and the size of the female pelvis and capacity of the outlet, may to some of your readers appear rather singular, and many others may feel and think as I do, that it is also singular, when we consider and observe the vast difference in the size and development of this important and useful member of the human frame, in those men we meet with who are daily engaged in practising in that delicate and peculiar branch of our profession. It is extraordinary that it has never attracted their attention, or been noticed by the various teachers and professors of the obstetric science or the general practitioners of midwifery; and it seems also to be unobserved by all authors on this subject, for we have published 